\title{
Foreshortening and the perception of parallel projections
}

\author{
ANDREA L. NICHOLLS \\ McMaster University, Hamilton, Ontario, Canada \\ and \\ JOHN M. KENNEDY \\ University of Toronto, Toronto, Ontario, Canada
}

\begin{abstract}
Does picture perception follow polar projective geometry? Parallel projection drawings, which are not produced by using rules of polar projection, are widely regarded as visually acceptable representations of three-dimensional (3-D) objects in free viewing. One explanation is that they are perceived by means of a system in which there is no foreshortening. If so, edges of a 3-D block in 1:1 proportions should be denoted by lines in 1:1 proportions on the picture surface. However, three experiments suggest that the perception of parallel projections of a block involves foreshortening. In Experiment 1, 90 subjects were shown a set of parallel projections of a cube, in which each drawing depicted three sides of the cube, drawn as a square with obliques-a frontal square with receding edges shown by parallel obliques of various lengths. The subjects preferred a drawing with a receding side length that was considerably foreshortened in relation to the front side. In Experiments 2 and 3, subjects viewed drawings of three blocks that differed in the ratios of the lengths of their receding edges to their frontal edges (1:1, 1:2, and 1:0.65). In Experiment 2, the subjects were shown square-with-obliques drawings of the three blocks with receding edges shown by parallel obliques of various lengths. Again, the subjects preferred drawings with a receding side that was foreshortened. In Experiment 3, the drawings showed two sides of a block. The receding dimension was drawn with parallel or converging lines. The preferred foreshortening was not a fixed ratio of the dimensions of the 3-D blocks. We suggest that square-with-obliques parallel projections showing cubes are taken by vision to be approximations to projections using foreshortening. We suggest also that as the line showing the receding edge elongates, foreshortening becomes less of a factor.
\end{abstract}

Parallel projection systems have been used extensively to depict three-dimensional (3-D) objects and scenes in free viewing. Early Greek, Roman, Byzantine, Persian, and Chinese art all provide instances of the use of parallel projection (Arnheim, 1974; Dubery \& Willats, 1983; Hagen, 1985, 1986). Research in drawing development also indicates that parallel projections are widespread. Many older children ( 10 years and above) and adults produce a square with parallel oblique lines when asked to draw a cube (Arnheim, 1974; Caron-Pargue, 1985; Cox, 1986; Freeman, 1980, 1986; Willats, 1981, 1984). But the widespread use of parallel projection to represent three dimensions suggests problems for theories which contend that the perception of depth in pictures follows the laws of polar projective geometry. Parallel projection draw-

\footnotetext{
We thank the Ontario Science Centre for providing invaluable testing facilities. We are grateful to Lauren Barnes and Richard MacLaren for their assistance with data collection. A. Nicholls is at the Schnurmacher Institute for Vision Research, SUNY/State College of Optometry, 100 East 24th St., New York, NY 10010. J. M. Kennedy is at the Department of Psychology, Life Sciences Division, University of Toronto/Scarborough College, 1265 Military Trail, Scarborough, ON, Canada M1C 1A4.
}

ings are not produced according to polar projection rules. Indeed, explanations for the success of parallel projection drawings are often couched in terms of the observer's knowledge about the structure and features of the 3-D object (Cox, 1986; Duthie, 1985; Freeman, 1986, 1987), rather than the optic projection from the picture. In the face of this knowledge-based view, we suggest that the perception of parallel projection drawings of cubes is related to how well they reenact what is projected from the 3-D object to a specific vantage point. In three experiments, we use one aspect of vantage-point geometry, foreshortening, to test the hypothesis that polar projective geometry is involved in the perception of parallel projections. We also suggest an important role for knowledgebased theory in the depiction of noncubic objects.

The geometrical rules for the construction of polar projection drawings assume an observer with his/her eye at a fixed position and fixed distance from the scene (Kubovy, 1986; Pirenne, 1970; White, 1967). This fixed vantagepoint assumption has several important implications. Only the faces of the 3-D object visible from a vantage point are represented in the drawing. Also, lines in the drawing that show receding parallel edges of the 3-D object converge, and, if they are extended, they meet at points 
termed vanishing points. Hence, measures of angles in a drawing may or may not be equal to the measures of corresponding angles in a 3-D object. For example, the $90^{\circ}$ angles in a 3-D cube may be depicted as more acute or obtuse in the drawing on the page, in keeping with the visual directions of the edges of the cube from the vantage point.

As $D$, the distance to the object from the vantage point, increases, $O$, the angle subtended by the object, decreases following a tangent law: $\tan O=H / D$, where $H$ is the height of the object. As the ties of a railroad track recede, they subtend smaller angles. A picture of the ties uses shorter lines-foreshortened lines-to indicate increase in distance. The tracks are said to converge. The picture can create the same pattern at its correct vantage point as the real scene.

The system of parallel projection, however, does not require a unique, fixed vantage point. Parallel projection drawings have no vanishing points, save at infinity. Lines representing receding edges of the object are shown as parallels. Also, some parallel projection drawings (e.g., cavalier oblique projection; see Dubery \& Willats, 1983) preserve proportions of line lengths, whether the edge they are showing is frontal or receding. That is, if two sides of an object have the same metric length, as in the $1: 1$ proportions of edges in a cube, they are represented by 1:1 proportions in the lines denoting them on the page (see Figure 1, top). There is no variation in line length to indicate the distance from the edge of the cube to the observer. We should point out that other types of parallel projection drawings may have lines of different lengths on the page, to show the receding sides of an object. Cabinet oblique projection, for example, uses half lengths to indicate receding sides (Dubery \& Willats, 1983). Notice that cabinet oblique projection preserves some of the parallels present in a 3-D cube, but also includes foreshortening, an aspect of vantage-point geometry (see Figure 1, bottom).
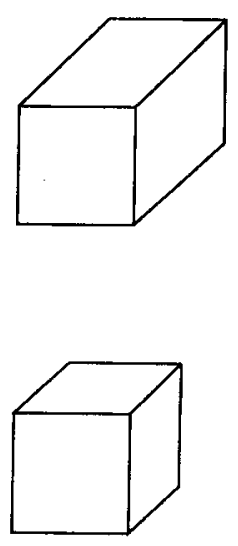

Figure 1. Two types of oblique projection in square-with-obliques drawings of cubes: cavalier oblique projection (top), and cabinet oblique projection.
In parallel projections, the fixed vantage-point assumption is not used to re-create the optic geometry projected by a 3-D object. Why, then, are parallel projections so widely advocated as acceptable and visually successful depictions of 3-D objects and scenes?

One explanation is that parallel projection drawings may be successful to the extent that they entail properties on the picture surface that concur with knowledge of 3-D object structure (Arnheim, 1974; Cox, 1986; Duthie, 1985; Freeman, 1986). Consider the cube drawings in Figure 1. They are of a type commonly produced by subjects asked to draw a cube. We will call them square-with-obliques drawings. A square represents a face of the cube parallel to the picture plane, and parallel obliques of various lengths are used to show receding sides of the cube. Arnheim (1974) describes this kind of drawing as looking "cube-like" because its properties on the picture surface preserve parallelism, an "essential objective property" of a 3-D cube.

Cox (1986) asked 26 undergraduates to draw a cube from memory. All produced a square-with-obliques version. She suggests that they may have found this to be a "good" representation of a cube because it preserves known "facts" about cubes. Even when adults were asked to draw a cube as they saw it from a specific vantage point (where only the top and front faces were visible), many of them used parallel lines to represent receding edges of the cube. Cox's explanation was that "their knowledge of invariant properties of the cube was so dominant that it interfered with or even prevented any attempt to capture the fleeting look of the cube from their particular arbitrary viewpoint"' (p. 345).

Freeman (1986) supported this knowledge-based interpretation of square-with-obliques drawings of cubes. He has described the square-with-obliques drawing as specifying "the structural description of a cube" without capturing its appearance (p. 317). He reasons that it is an acceptable representation of a cube because it is a compromise between appearance and structure. Freeman also considers aspects of the drawing that match those of a 3-D cube. For instance, he says that "all pairs of parallels are preserved" 'and that "side lengths are in constant proportion, as in a real cube" (pp. 317-318). Duthie (1985) has also described the square-with-obliques drawing as a compromise between knowledge and appearance. He says that it can "depict at least some right angles as right angles, and, all parallel lines as parallel and show three sides in correct relative size" (p. 110).

Unfortunately, although it is obvious that some properties of the square-with-obliques drawing correspond to the known structure of cubes, it is not certain that subjects use these features, or what might influence possible compromises, or what creates the appearance of a 3-D cube. The parallel lines in the drawing could represent a known relationship between the sides of a cube, or they could create the visual appearance of a real cube. Similarly, the constant proportion of side lengths might represent the proportions of sides as we know them to be 
in a real cube, or they might create a percept of sides as they look in a real cube. Also, it is not obvious whether angles on the page match what we know about the properties and features of a cube, or result from vantage-point geometry and actually help re-create the appearance of a cube.

Interestingly, Cox (1989) and Freeman (1986) agree that the obliques in square-with-obliques drawings may reflect vantage-point geometry. Cox (1989) conjectures that the oblique lines in the square-with-obliques drawing are included to influence the appearance of the depicted cube. Freeman (1986) points out that the oblique line may be used to represent the direction of an edge of a 3-D cube from the viewer. Similarly, Nicholls and Kennedy (1992), in a study of drawings of cubes produced by subjects of various ages (5-year-olds to adults), suggested that older children and adults may produce squarewith-obliques drawings while trying to match the projections of parts of the 3-D cube to a vantage point. Nicholls and Kennedy found that the use of foreshortening increased with age. If the use of parallel projection is influenced by vantage-point geometry, nonfrontal edges should be shown with foreshortened lines on the page.

We examined examples published by theorists writing about cube drawings, especially those from theorists writing about a knowledge-based account (Arnheim, 1974; Caron-Pargue, 1985; Cox, 1986; Deregowski \& Strang, 1986; Freeman, 1986, 1987; Mitchelmore, 1987; Moore, 1987; Phillips, Hobbs, \& Pratt, 1978; Willats, 1984). Only 1 of 10 cube figures, that of Willats (1984), had the front-line-to-side-line ratio of $1: 1$ that a knowledge-based view would suggest. Both Arnheim (1974) and Cox (1986) showed examples of figures with a 1:0.72 ratio. Freeman's $(1986,1987)$ figures have ratios of 1:0.75. These ratios illustrate our point. Instead of depicting proportions that match the known 1:1 proportions of a 3-D cube, the figures contain foreshortening. The use of foreshortening may engender the visual appearance of $1: 1$ proportions for a 3-D cube and reflect the influence of vantagepoint geometry.

In Experiment 1, we tested preferences for square-withobliques drawings of cubes showing different degrees of foreshortening. The stimuli were chosen on the basis of a preliminary study with 67 volunteers (ranging in age from 9 to 66) at the Ontario Science Centre, who were shown nine square-with-obliques drawings of cubes with front-line-to-side-line foreshortening ratios ranging from 1:0.32 to 1:1.5 (Figure 2). In the preliminary study, three sets of nine were used, which differed in the angle at which the front horizontal line of the square met the oblique line representing the receding side $\left(30^{\circ}, 45^{\circ}\right.$, and $\left.60^{\circ}\right)$.

While seated at a table, the subjects in the preliminary study were given a booklet containing the instructions and stimuli to examine in free viewing. They were asked to circle the best drawing of a cube from the set of nine square-with-obliques figures. The square-with-obliques figure with a front-line-to-side-line ratio of 1:0.62 was preferred by over half the subjects (Table 1), irrespective
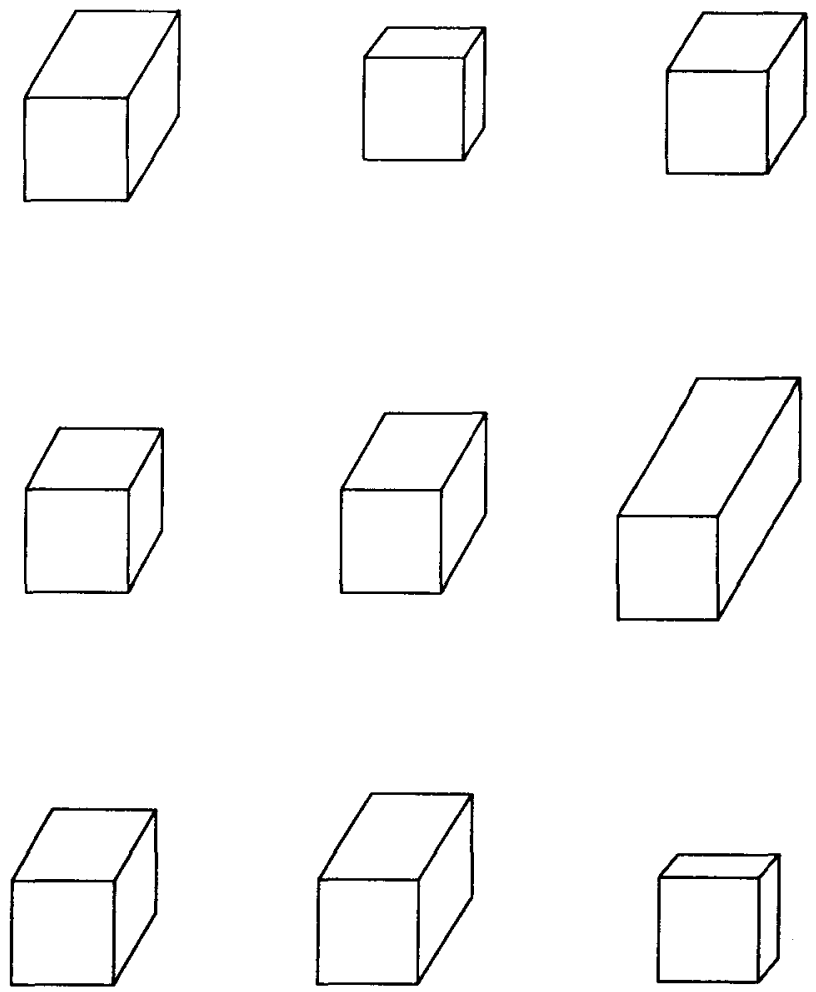

Figure 2. Nine square-with-obliques drawings, varying in foreshortening ratio, used in the preliminary experiment.

of the angle at which the oblique line met the horizontal. The foreshortening ratios for the four drawings selected most often by subjects were used in Experiment 1.

\section{EXPERIMENT 1}

\section{Method}

Subjects. The 90 subjects ranging in age from 14 to 55 volunteered to participate at the Ontario Science Centre. All had normal or corrected-to-normal vision. Sixty-six of the participants were shown Set 1 drawings, and 24 participants were shown a similar, redrawn set (Set 2). Two sets were used to control for any minor variations in the drawings that might have arisen in sketching with a ruler.

Stimuli. The stimuli consisted of square-with-obliques line drawings varying in the length of the oblique lines showing the receding dimension (Figure 3 ). The front square in each drawing measured $2.2 \times 2.2 \mathrm{~cm}$. Each subject was shown four drawings, with foreshortening ratios of 1:0.38, 1:0.65, 1:0.73, and 1:0.81. (These ratios varied slightly from their counterparts in the preliminary experiment, because the drawings were redrawn at a new size, with thinner lines.)

Three different versions of the drawings were produced. In one version, the oblique lines met the horizontal at $45^{\circ}$. In a second version, this angle was $30^{\circ}$, and in a third, it was $60^{\circ}$. There were four randomized orders of the drawings for each version. A second set of the 12 drawings (Set 2) was created to verify the results, since small deviations in line length (about $0.1 \mathrm{~cm}$ ) occur as a result of the drawing technique.

Procedure. Participants were tested individually. Each subject was seated at a table and given a small booklet containing the in- 
Table 1

Preliminary Study for Experiment 1: Number of Subjects Selecting Each Drawing as Best

\begin{tabular}{lccccccccccc}
\multicolumn{8}{c}{ Number of Subjects Selecting Each Drawing as Best } \\
\hline $\begin{array}{l}\text { Angle of } \\
\text { Oblique }\end{array}$ & 0.32 & 0.36 & 0.62 & 0.77 & 0.81 & 0.91 & 1.0 & 1.1 & 1.5 \\
\hline $30^{\circ}$ & 0 & 1 & 12 & 5 & 2 & 0 & 0 & 0 & 0 \\
$45^{\circ}$ & 1 & 2 & 12 & 2 & 6 & 0 & 1 & 0 & 0 \\
$60^{\circ}$ & 0 & 0 & 12 & 5 & 6 & 0 & 0 & 0 & 0 \\
Total & 1 & 3 & 36 & 12 & 14 & 0 & 1 & 0 & 0 \\
\hline
\end{tabular}

Note-In a few cases, the ratio varied slightly across the three angles because of variations in line lengths of $0.1 \mathrm{~cm}$ due to drawing. In two cases, there were errors in drawing. One case was the $1: 1.1$ ratio figure with the $45^{\circ}$ angle. The receding line was inadvertently extended by $0.4 \mathrm{~cm}$, creating a ratio of $1: 1.27$. The second case was the $1: 1.5$ ratio drawing for the $60^{\circ}$ figure. Here the receding line was inadvertently shortened by $0.4 \mathrm{~cm}$, creating a ratio of $1: 1.35$. However. it is unlikely that these errors had much bearing on the results, since no subjects selected ratios greater than $1: 1$.

structions and the stimuli, along with a pencil. Their task was to select the best drawing of a cube and write "best" under the drawing. Then each subject was asked to decide which of three remaining figures was "second best" and finally to select the "worst" drawing of a cube. These written responses were assigned numerical rankings for data analysis.

\section{Results}

Table 2 presents the mean of the rankings assigned to the four drawings for the three angles of obliqueness. For all three angles, the ratio receiving the highest mean ranking was $1: 0.65$. The $1: 0.73$ ratio drawing always received the second highest ranking. The $1: 0.38$ ratio drawing had the lowest mean ranking for $30^{\circ}$ and $45^{\circ}$, while the 1:0.81 ratio had the lowest mean for $60^{\circ}$. A Friedman two-way analysis of variance showed the ratio effect to be significant $\left[\chi^{2}(4, N=3)=8.2, p<.02\right]$.

Log-linear analyses were conducted to determine effects of set and obliqueness angle on the ratio selected as best by each subject. The models of interest were those testing the effect of ratio, the effect of set on ratio selection, the effect of obliqueness angle on ratio selection,
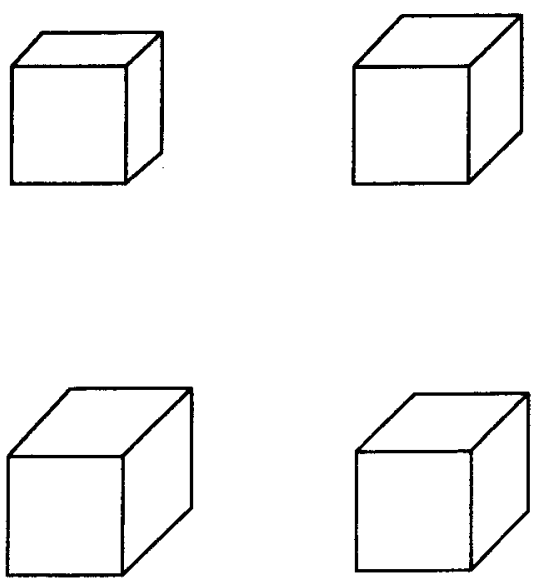

Figure 3. Four square-with-obliques drawings, varying in foreshortening ratio, used in Experiment 1. and the interaction of set and obliqueness angle (see Green, 1988). The model containing the ratio variable alone provided the best fit to the data $\left[G^{2}(15, N=90)=\right.$ $13.82, p<.54]$. Including the factors of set or obliqueness angle or their interaction did not significantly improve model fit.

\section{Discussion}

The subjects greatly preferred the square with obliques with the 1:0.65 ratio as the best drawing of a cube. They ranked the drawing with the ratio closest to $1: 1$, the $1: 0.81$ ratio drawing, as the third best. Clearly, the subjects preferred a parallel projection drawing that had considerable foreshortening, although not as much as in the 1:0.38 square-with-obliques drawing, which was consistently ranked worst.

It is relevant to note here that the subjects in the preliminary study did not have the opportunity to view drawings exhibiting foreshortening ratios between 1:0.36 (1:0.38) and 1:0.68 (1:0.65). Thus, we cannot say that the preferred 1:0.65 ratio is the single "best" ratio (though Cutting, 1986, has noted that drawings with cabinet oblique parallel projection, which provide a 1:0.50 foreshortening ratio, "often look too thin'). The evidence here does confirm that the 1:1 ratio, which would be expected from a concern for knowledge of cube properties, is not highly preferred. Instead, in a square-with-obliques drawing that was most successful, the receding sides had some foreshortening.

One possible explanation for these findings is that the preferred foreshortening ratio in the square-with-obliques drawing reflects an illusion suffered by any obliques (e.g., the horizontal-vertical illusion). That is, the oblique lines in the 1:0.65 ratio might actually look equal to the horizontal lines, as lengths of lines on a page. However, it seems likely to us that the lines showing the receding edges do look noticeably shorter than those showing the sides of the square, if one tries to see them simply as lines on the page.

We tested this possibility with 12 undergraduates at McMaster University in a study involving magnitude es- 
Table 2

Mean Rankings for Drawings Varying in Foreshortening Ratio for Experiment 1

\begin{tabular}{ccccc}
\hline $\begin{array}{c}\text { Angle of } \\
\text { Oblique }\end{array}$ & \multicolumn{5}{c}{ Foreshortening Ratio } \\
\cline { 2 - 5 } & $\mathbf{0 . 3 8}$ & 0.65 & 0.73 & 0.81 \\
\hline $30^{\circ}$ & 1.68 & 3.35 & 3.0 & 2.06 \\
$45^{\circ}$ & 1.79 & 3.37 & 2.80 & 2.06 \\
$60^{\circ}$ & 2.02 & 3.23 & 3.01 & 1.75 \\
\hline
\end{tabular}

timation. These subjects saw six line drawings, each of which contained a horizontal line measuring $3.2 \mathrm{~cm}$ and an oblique line that met the horizontal at $45^{\circ}$. Three different figures were used (Figure 4). One was a squarewith-obliques figure of a cube or box, to assess the perception of the lines in pictorial space. Two additional patterns containing the horizontal and oblique lines were included, to test the perception of the lines on the page. One was the hexagonal outline of the cube figure. The other also maintained the hexagonal outline of the cube figure, but included a cross inside. For each figure, two versions were produced. One provided a horizontal-tooblique line ratio of 1:0.65, and a second had a ratio of $1: 1$

The six line drawings were shown individually on vertical cards at the subjects' eye level, $1200 \mathrm{~cm}$ from where they were seated. There were no restrictions on head or
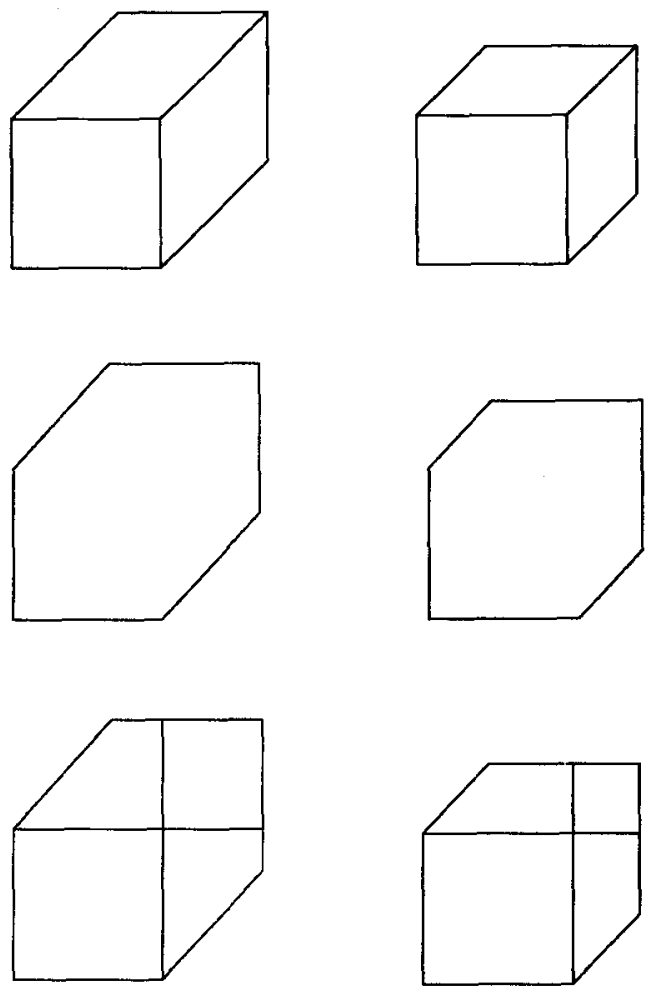

Figure 4. Square-with-obliques 1:1 ratio (top left), square-withobliques 1:0.65 ratio (top right), hexagon 1:1 ratio (center left), hexagon 1:0.65 ratio (center right), cross-and-hexagon 1:1 ratio (bottom left), and cross-and-hexagon 1:0.65 ratio (bottom right). eye movements. The order of presentation of the six figures was counterbalanced. For each display, the subjects were told that the horizontal line was 10 units long, and they were asked how long the oblique line was in comparison.

A 3 (figure type) $\times 2$ (ratio) analysis of variance for a repeated measures design yielded main effects of figure type $\left[F(2,22)=15.69, M S_{\mathrm{e}}=3.91, p<.001\right]$ and ratio $\left[F(1,11)=89.76, M S_{\mathrm{e}}=3.12, p<.001\right]$. There were no significant interactions. For the hexagon and the cross-and-hexagon figures, estimates of the line length for both the 1:0.65 ratio figure and the 1:1 figure were quite accurate. The mean ratios for the 1:0.65 figure were 1:0.68 for the hexagon and 1:0.73 for the cross and hexagon. For the 1:1 figure, the mean ratios were $1: 1$ for the hexagon and $1: 1.1$ for the cross and hexagon. These findings suggest that the subjects could accurately assess the length of the oblique line in relation to the horizontal on the page. However, for the square-with-obliques figure, the mean ratios were 1:0.91 for the 1:0.65 figure, and $1: 1.39$ for the $1: 1$ figure. That is, the subjects may indeed have perceived the 1:0.65 square-with-obliques drawing as representing the appearance of a cube with equal-measure sides in pictorial space. The subjects presumably saw the $1: 1$ equal length square-with-obliques figure as looking "too long" to represent the appearance of a 3-D cube. No subjects judged the ratio in the 1:1 square-with-obliques figure to be $1: 1$ or less. These findings provide some evidence against the notion that the preferred ratio is due to a geometrical illusion, such as the horizontal-vertical illusion.

We suggest that the 1:0.65 ratio reflects a concern for how well the parallel projection drawing reenacts what is projected from a 3-D cube to a vantage point. The length of the lines showing the receding sides of the cube in the drawing is meant to produce the appearance in depth of the length of the sides in a 3-D cube. The implication is that for parallel projection, vision does not rely entirely on shapes and sizes on the picture surface: some visual projective geometry and apparent depth seem to be involved, not knowledge about matching the length and proportions of parts of the object with the length and proportions of parts in the drawing on the page.

We are not certain why 1:0.65 (or thereabouts) is the preferred foreshortening for a parallel projection of a cube. However, a cube is a highly familiar object, and subjects may have had access to a stereotypical or canonical representation of a cube, which involved taking a specific vantage point. One way to test this specific vantagepoint notion is to obtain judgments about foreshortening for objects that are not cubes.

In Experiment 2, subjects made judgments about one of three blocks, each with a square "front" face. One was a regular cube. In a second block, the length of the receding side was $65 \%$ of the length of the frontal square. In the third block, the length of the receding side was twice the length of the front. Our primary goal was to examine whether the subjects would prefer square-with-obliques drawings of the three blocks that contained foreshortening. The use of three different lengths of receding dimen- 

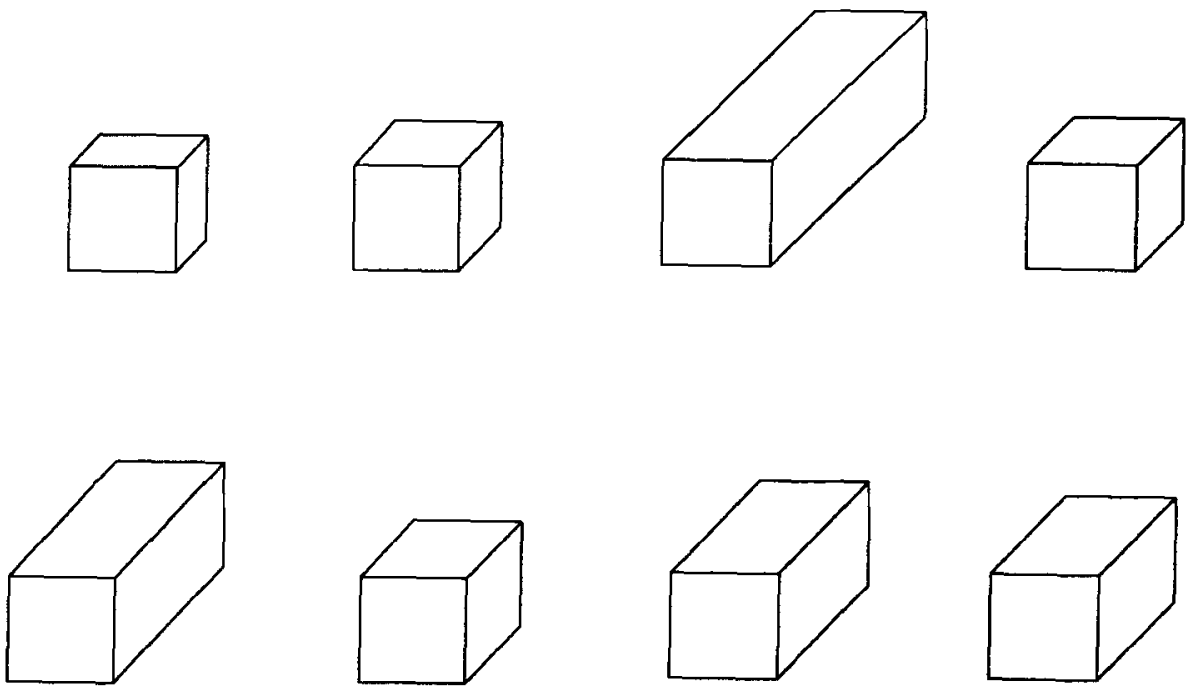

Figure 5. Eight square-with-obliques drawings of the cubic block, varying in foreshortening ratio, used in Experiment 2.

sion for the 3-D blocks also permitted us to determine whether the preferred foreshortening ratio was a constant proportion of the length of the receding side in the object, or whether the preferred foreshortening ratio was related to the length of the receding side.

\section{EXPERIMENT 2}

\section{Method}

Subjects. One hundred and forty-four children and adults, ranging in age from 8 to 50 years, volunteered to participate at the Ontario Science Centre. These subjects all had normal or correctedto-normal vision.

Stimuli. The subjects examined one of three wooden blocks. One was a regular cube (1:1 block), whose edges all were equal (4.2 $\times 4.2 \times 4.2 \mathrm{~cm}$ ). A second had the same width and height as the cube, but extended to twice its length $(4.2 \times 4.2 \times 8.4 \mathrm{~cm})$. A third block was truncated, measuring $4.2 \times 4.2 \times 2.73 \mathrm{~cm}$, with the truncated dimension reduced to $65 \%$ of the other two.

For each of the three blocks, a set of eight parallel projection line drawings was produced. Figure 5 shows the eight drawings for the regular cube (1:1 block). Each drawing contained a square, and the drawings varied in the lengths of the oblique lines showing the receding sides. For each block, eight proportions of front line to receding line were used: 1:0.4, 1:0.5, 1:0.65, 1:0.8, 1:1, 1:1.2, $1: 1.5$, and $1: 2$. The eight drawings for each block were presented in eight randomized orders on the page, counterbalanced across subjects.

Procedure. The subjects were seated at a table and individually shown one of the three wooden blocks. The relative lengths of the sides were pointed out: "Notice the length of this side of the block [the long or short side] in relation to this side of the block [the square side]." The block was given to the subject to inspect. Then the experimenter placed the block with the square facing the subject, at a distance of $26 \mathrm{~cm}$ on the table along a normal from the block face to the table edge and $16 \mathrm{~cm}$ to the left of the straight ahead. From this position, the subject could view the front face and two receding sides of the block. The subjects were told, "I'll set the block here so you can look at it again if you want to."
A page with the set of eight drawings of the block was placed on the table in front of each subject. They were asked to select the best drawing of the block.

\section{Results}

Table 3 shows the number of subjects selecting each foreshortening ratio for each block. In the table and subsequent discussion, ratios of lines are expressed relative to the actual proportions of the edges in the block. That is, the figure with the 1:0.65 ratio for the truncated block (1:0.65) had lines in a 1:0.42 proportion on the page; the 1:0.65 figure for the cubic block (1:1) had lines in a 1:0.65 proportion on the page; and the figure with the 1:0.65 ratio for the extended block (1:2) had lines in a $1: 1.3$ proportion on the page.

For the truncated block $(1: 0.65), 42 \%$ of the subjects preferred the 1:0.65 ratio. (That is, they preferred the drawing with lines in a 1:0.42 ratio on the page.) The most frequently chosen ratio for the cubic block $(1: 1)$ was also $1: 0.65$, by $33 \%$ of the subjects. For the extended block (1:2), $46 \%$ of the subjects preferred a foreshortening ratio of 1:0.8. (That is, the actual ratio of lengths on the page was $1: 1.6$.) Note that the preferred ratio for each block was less than the measured proportion of the sides in the block. The subjects evidently preferred depictions

Table 3

Number of Subjects Selecting Each Drawing (Foreshortening Ratio) for Each of the Three Blocks

\begin{tabular}{lcrrrrrrr}
\hline & \multicolumn{8}{c}{ Foreshortening Ratio } \\
\cline { 2 - 10 } \multicolumn{1}{c}{ Block } & 0.4 & 0.5 & 0.65 & 0.8 & 1.0 & 1.2 & 1.5 & 2.0 \\
Truncated & 3 & 14 & 20 & 6 & 1 & 1 & 2 & 1 \\
Cubic & 7 & 10 & 16 & 11 & 4 & 0 & 0 & 0 \\
Extended & 0 & 1 & 16 & 22 & 8 & 0 & 0 & 1 \\
\hline
\end{tabular}


of the blocks with receding sides that were foreshortened relative to their actual proportions. Indeed, for the cubic block, no subjects selected ratios where the proportion of the receding side as opposed to the front side was greater than in the actual block; only 4 subjects did so for the truncated block, and only 1 for the extended block.

Log-linear analyses were performed to assess the effects of block length and age on the selection of the foreshortening ratio. The subjects were divided into three age groups, 8-10,11-13, and 14 and older, with 48 subjects in each group. The models of interest were those testing the effect of block length on foreshortening ratio, the effect of age on foreshortening ratio, and the interaction of block length and age (see Green, 1988). The model containing the main effect of block length $\left[G^{2}(42, N=\right.$ $192)=37.57, p<.66$ ] provided the best fit to the data, suggesting that the preferred foreshortening ratio depended on the length of the receding side of the block. Including age effects did not improve the model fit.

While 1:0.65 is the mode for the truncated block (1:0.65), more subjects (17) selected ratios below 0.65 in two categories $(0.4$ and 0.5$)$ than above (11 subjects, in 5 categories from 0.8 to 2). A similar bias is evident in judgments for the extended block. Preferences for the cubic block (1:1) were more evenly balanced above (15 subjects) and below (17 subjects) the mode of 1:0.65.

\section{Discussion}

For all three blocks, the subjects preferred square-withobliques drawings that depicted front-to-side proportions as foreshortened relative to their actual proportions in the 3-D block.

The preference for foreshortening in the parallel projection drawings of the three blocks can be related to the "natural perspective" projected to a vantage point from the real 3-D object. In natural perspective, a cube's front face always subtends a greater angle than does the side face. Presumably, the subjects took the square in the square-with-obliques drawing to be the front face.

We should point out that in artificial perspective, when a picture of an object is present-that is, when a picture surface is placed between the object and the vantage point - a far wider set of projected proportions from the depicted convergent "side" and square "front" faces is possible than under natural perspective. In many of these projections, the convergent part of the picture representing a side face is larger (and can project a much larger angle) than the square part of the picture representing the front face (see, e.g., Pirenne, 1970, Figures 9.11B and 9.11C). Since vision prefers a well-marked degree of foreshortening, vision does not use a huge range of artificial perspective ratios. We will return to this later.

Square-with-obliques showing three faces are common drawings of cubes. Cubes can also be drawn with only two faces showing, in parallel or convergent perspective. Dubery and Willats (1983) have pointed out one variant of this style, in which the front of a cube is shown as a square and verticals are used to show the cube's top face-a style called vertical oblique. In comparison with the style used in the present Experiments 1 and 2, vertical oblique is uncommon in drawings of cubes made by children and adults (Nicholls \& Kennedy, 1992). It may be used to test for the use of foreshortening in fairly unfamiliar kinds of parallel projection drawings, and to compare judgments of parallel and convergent stimuli.

\section{EXPERIMENT 3}

\section{Method}

Subjects. Seventy-two undergraduate students participated as part of course credit in an introductory psychology class at McMaster University. All had normal or corrected-to-normal vision.

Stimuli. The subjects were shown each of the three blocks used in Experiment 1-the truncated block (1:0.65), the cubic block (1:1), and the extended block $(1: 2)$-individually. Two sets of six line drawings were produced for each block. In both sets, a square was drawn to show a side of the block facing the observer. In the parallel projection set (Figure 6), the receding top of each block was represented with vertical lines. Figure 7 shows the converging projection set, in which the receding top of each block was represented with converging, oblique lines. The angle between each of the oblique lines and the line showing the top edge of the block's front face was $85^{\circ}$ in each drawing. For both the parallel projection set and the converging projection set, six drawings were produced. The ratios of the line for the receding edge to the line showing the front edge of the block were 1:0.4, 1:0.5, 1:0.65, 1:0.8, and 1:1. Again, the $1: 0.8$ ratio for the truncated block $(1: 0.65)$ means that the lines were in a 1:0.52 proportion on the page. The same ratio for the extended block (1:2) was shown by using lines in a $1: 1.6$ proportion on the page. The order of presentation of the six drawings for each block was counterbalanced across subjects.

Procedure. The subjects inspected one of the three blocks and were instructed to notice the length of the receding side relative
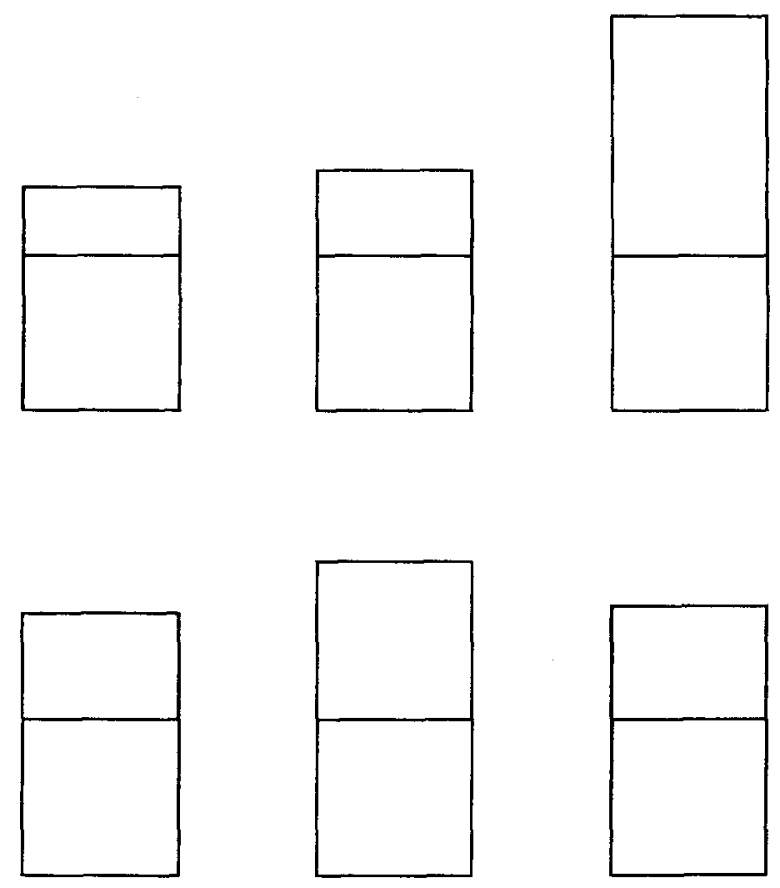

Figure 6. Parallel versions of six two-sided drawings of the cubic block, varying in the length of the vertical, parallel lines showing the receding side. 

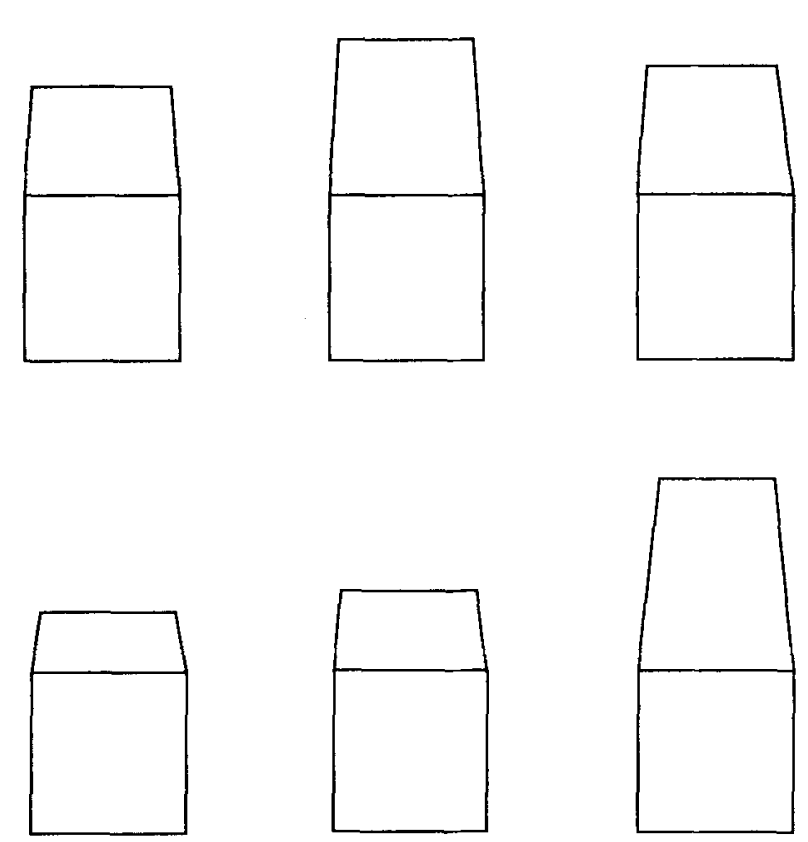

Figure 7. Convergent versions of six two-sided drawings of the cubic block, varying in the length of the converging, oblique lines showing the receding side.

to the length of the front side. The block was then placed $40 \mathrm{~cm}$ directly in front of the subject on the table top, with the front square and top of the block available to the observer. Half the subjects selected the best drawing of the block from the set of six parallel projection drawings, and half made their selection from the converging projection set. The procedure was then repeated for each of the other two blocks, with the same projection set.

\section{Results}

Table 4 shows the number of subjects selecting each foreshortening ratio for the parallel and convergent projection sets for each of the three blocks. The preferred ratios for the parallel projection drawings were significantly different from those for the converging projection drawings $\left[\chi^{2}(4, N=72)=32.79, p<.001\right]$. In general, the preferred ratios for the parallel drawings were higher than those for the converging drawings. For par-

Table 4

Number of Subjects Selecting Each Drawing (Foreshortening Ratio) for the Parallel Projection and Converging Projection Sets for Each Block

\begin{tabular}{lrrrrrr}
\hline & \multicolumn{6}{c}{ Foreshortening Ratio } \\
\cline { 2 - 5 } \multicolumn{1}{c}{ Block } & 0.4 & 0.5 & 0.65 & 0.8 & 1.0 & 1.2 \\
Truncated & 1 & 1 & 10 & 20 & 4 & 0 \\
Cubic & 0 & 1 & 5 & 18 & 12 & 0 \\
Extended & 0 & 0 & 2 & 14 & 20 & 0 \\
& \multicolumn{7}{c}{ Parallel } \\
Truncated & 3 & 22 & 7 & 4 & 0 & 0 \\
Cubic & 3 & 13 & 10 & 9 & 1 & 0 \\
Extended & 1 & 7 & 16 & 10 & 2 & 0 \\
\hline
\end{tabular}

allel drawings of the truncated block (1:0.65) $55 \%$ of the subjects preferred the 1:0.8 foreshortening ratio, whereas for the convergent drawings of the same block, the majority (61\%) preferred the 1:0.5 ratio. For the cube $(1: 1), 50 \%$ of the subjects chose the 1:0.8 ratio for the parallel drawings, and $36 \%$ chose the 1:0.5 ratio for the convergent drawings. The preferred ratio for the parallel drawing of the extended block (1:2) was $1: 1$ (55\% of the subjects), but, for the convergent drawing, the preferred ratio was 1:0.65 (44\% of the subjects). No subjects chose foreshortening ratios greater than $1: 1$ for any of the three blocks in the parallel or convergent versions.

Each of the two drawing types (parallel and convergent) can also be considered separately for the within-subjects effect of block length. A Friedman two-way analysis of variance on the parallel drawings showed that rankings for the three different blocks were significantly different from each other $\left[\chi^{2}(2, N=36)=17.01, p<.01\right]$. Rankings for the three blocks were also significantly different for subjects who saw the convergent drawings $\left[\chi^{2}(2, N=\right.$ $36)=10.51, p<.01]$. While the mode of $1: 0.8$ for the 1:0.65 block was flanked by more subjects selecting a lower ratio (12 subjects) than a higher ratio (4) in the case of the parallel projection, in the case of the cube $(1: 1$ block) the mode was flanked by more subjects preferring a higher ratio (12 subjects) than a lower ratio (6).

\section{Discussion}

Experiment 3 was designed to test whether subjects preferred foreshortening ratios in parallel projections of blocks that used parallel vertical lines, rather than oblique lines, to show the receding sides. Our findings suggest that they do. For both the truncated block and the cubic block, the foreshortening ratio preferred by a large number of subjects was 1:0.8. However, for the extended block, the preferred ratio was 1:1 (though no subjects preferred a ratio above $1: 1$ ).

It is of interest that there were differences in the preferred foreshortening ratios for the parallel and convergent versions for each block. Foreshortening ratios for the convergent versions of the three blocks were substantially smaller than their parallel projection counterparts1:0.5 for the truncated and cubic blocks (vs. 1:0.8), and 1:0.65 for the extended block (vs. 1:1). We suggest that the oblique line is more effective than the parallel vertical lines in creating apparent depth.

\section{GENERAL DISCUSSION}

Why is a parallel projection drawing subject to foreshortening criteria in free viewing? And why does the preferred foreshortening change as the block elongates?

Hagen $(1985,1986)$ suggests that parallel perspective is taken by vision to be a special kind of polar perspective. In parallel projection drawings, the 3-D object's location is so far away that light is projected to the vantage point in parallels. Although in theory a substantial object must be located at an infinite distance for reflected light to project as parallel, Hagen (1985) suggests that in prac- 
tice, for vision, optical infinity is much closer. She argues that a vantage point "assumed at a distance of 40 times the size of the object is effectively at optical infinity" (p. 60). That is, parallel projection drawings may be taken by vision to be extreme examples of the system of polar projection.

Consider a block held at arm's length and eye level, with its front face vertical. If the arm moves down to waist level, convergence of the sides of the top decreases (for a vertical picture plane). At the same time, the top side of the block projects a larger and larger angle to the vantage point. Note that as the convergence decreases, the projected ratio of the front to the side edge increases. This is precisely the relationship that the preferences in Experiment 3 follow. Parallel projection drawings require ratios closer to $1: 1$ than do convergent drawings, for a given block.

Why do the preferred foreshortening ratios vary with the length of the receding side of the block? A squarewith-obliques drawing shows a front face and a side face. It indicates that the block is not placed straight aheadthat is, with the vantage point on the normal from the middle of the front face. Rather, the vantage-point $P$ is somewhat to one side of the front face-a location where the side face is not occluded. A cube, with each edge of length 1 , may project 1:0.65 to a picture plane parallel to the front face, with vantage point $P$. Imagine that the cube is now doubled by adding another cube to the rear face. What will be the projection of this addition?

The answer is that the new addition will not require that the projection double. The projection will increase, but less than an additional 1:0.65. Notice that if the cube were to extend gradually back to infinity, it is a fundamental principle of polar perspective that the projection would increase, but at an ever decreasing rate, to a vanishing point at the foot of the normal from the vantage point to the picture surface.

The evidence from Experiments 1, 2, and 3 is that subjects violate this fundamental principle of polar perspective. Subjects use 0.65 foreshortening or more for cubes and truncated blocks, but less and less foreshortening (from 1:0.8 all the way to 1:1) for longer blocks.

It seems likely that subjects are using two principles. The selection between the principles depends on the proportions of the 3-D block. With shorter blocks, subjects' judgments involve foreshortening. Foreshortening judgments could stem from percepts of depicted objects, seen in depth, meaning that the features on the page stimulate a percept of an object in a pictorial 3-D world. The characteristics of that perceived object, not the features on the page directly, govern the subjects' judgments. Short lines on the page are seen "in depth," as being longer than they are on the page. With longer blocks, subjects' judgments are based on a scan of features on the page. A line $L$ on the page is assessed as depicting an edge of the same length as a line $L$ of the same length but at an oblique to $L$.

The reason for the subjects' shift in criteria as blocks elongate could be that short blocks depicted with foreshortening in parallel perspective are quite similar to con- vergent drawings, for objects with a small angular subtense, as Hagen (1986) noted. With longer blocks, it is possible to have stricter criteria for parallels. If so, only shorter blocks are deemed to be shown using convergence, and foreshortening is relevant.

A curious implication of our interpretation here is that Arnheim, Cox, Duthie, and Freeman are incorrect as far as the object of their discussions - a cube - is concerned. Their knowledge-based thesis actually catches hold with longer blocks. In Experiment 3, the modal preference was a 1:1 ratio with a 1:2 block, with lines for the receding side stretching vertically, although with a cube as the target block the subjects preferred 1:0.8.

A very important result in Experiment 3 is the shift in preferences that occurred with convergent obliques. These increased from 1:0.5 for the truncated block to 1:0.65 for the cube. The principle revealed in Experiment 2's results helps explain demonstrations by Pirenne (1970, Figures 9.11B and 9.11C). Pirenne's drawings, shown in Figure 8, represent cubes with two or three faces confronting the viewer, with frontal squares, parallelograms, rectangles, and trapezoids (see also Kubovy, 1986). The
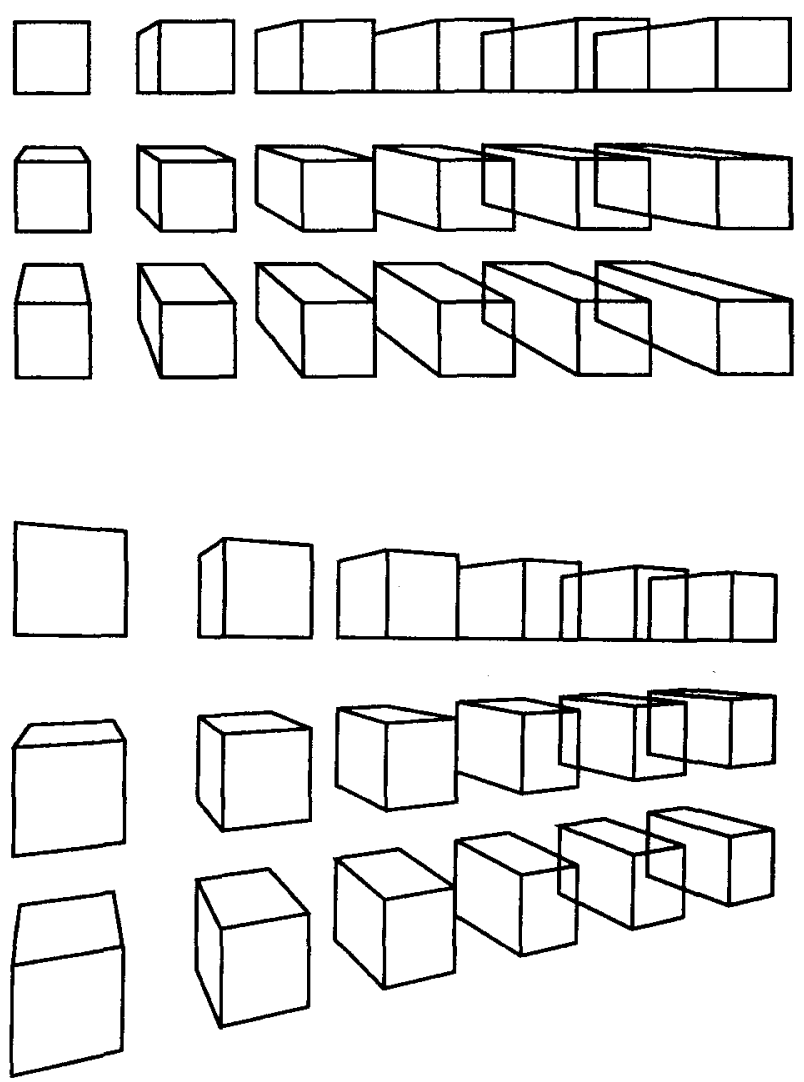

Figure 8. A central projection of a set of 18 cubes with the picture plane parallel to the front faces of the cubes (top) and a central projection of the same cubes with the picture plane set at a slant of $15^{\circ}$ to the plane containing the front cube faces. From Optics, Painting and Photography (p. 127) by M. H. Pirenne, 1970, Cambridge: Cambridge University Press. Copyright 1970 by Cambridge University Press. Adapted by permission. 

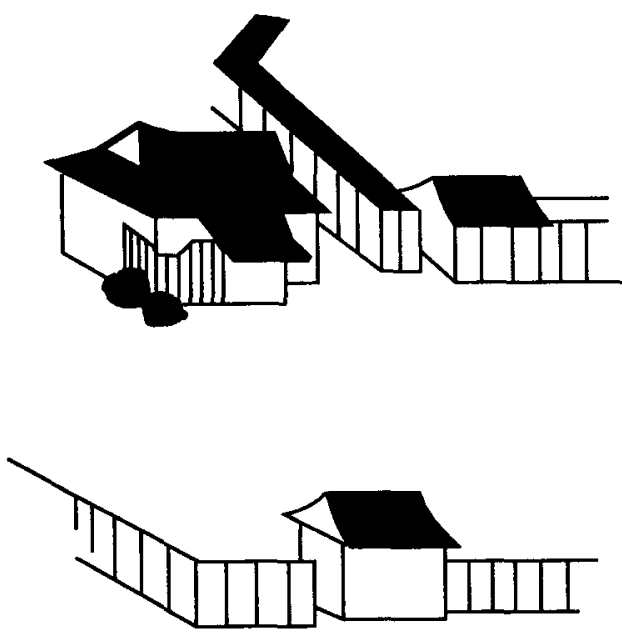

Figure 9. Based on Scenes from the Life of Gensei Shonin (No. 49.92), Seattle Art Museum, Eugene Fuller Memorial Collection. Adapted by permission.

drawings are in perfect perspective, but in free viewing most of them appear to represent extended blocks, not cubes. The farther the cube is set from the normal to the vantage point to the picture plane, the less acceptable is the resulting drawing in free viewing. Experiment 2 indicates that once the length of the line depicting a receding side edge of the cube approaches equality with the line depicting a frontal edge, in a square-with-obliques drawing, it will seem to depict the edge of an extended block. Cubes drawn as if set far from the normal to the picture plane have longer lines depicting receding side edges than cubes set close to the normal. The lines get longer the farther the cube is set from the normal, and the length approaches infinity in the extreme.

Experiment 3 reveals that for a given degree of convergence, vision prefers less foreshortening as the size of the block increases. This means that the longer the line for the side, in proportion to the front face, the stronger the tendency to take the proportions on the page as indicating the length of the edge in the 3-D object. It is little wonder that most of Pirenne's projections of cubes off the normal to the picture plane fail to be acceptable as depictions of cubes. Despite their geometrical precision, in free viewing the lengths and proportions of the lines on the page are evident.

As a corollary, we should note that art from Japan and China, for example, often involves large landscapes painted in parallel perspective (see Figure 9). Vision seems to use the actual proportions on the painted surface as a guide to the actual proportions of the depicted objects. Experiments 1 and 2 suggest that long receding walls are being taken to be equal in size to frontal walls, when they are depicted by lines of equal length in this kind of landscape depiction.

In summary, in free viewing, foreshortening is relevant visually for parallel perspective drawings with reced- ing sides of a block shown by lines shorter than lines showing frontal sides, as in polar (convergent) perspective. As the sides of the block elongate, less foreshortening is required on the page for parallel perspective drawings. The reduction in the use of foreshortening is also present in the viewing of polar perspective drawings as the side lines elongate.

\section{REFERENCES}

ARNHeIm, R. (1974). Art and visual perception (2nd ed.). Berkeley \& Los Angeles: University of California Press.

Caron-Pargue, J. (1985). Le dessin du cube chez l'enfant. New York: Peter Lang.

Cox, M. V. (1986). Cubes are difficult things to draw. British Journal of Developmental Psychology, 4, 341-345.

Cox, M. V. (1989). Knowledge and appearance in children's pictorial representations. Educational Psychology, 9, 15-25.

Cutting, J. E. (1986). Perception with an eye for motion. Cambridge, MA: MIT Press.

Deregowski, J. B., \& Strang, P. (1986). On the drawing of a cube and its derivatives. British Joumal of Developmental Psychology, 4, 323-330.

DUbERy, F., \& Willats, J. (1983). Perspective and other drawing systems. London: Herbert Press.

DUTHIE, R. K. (1985). The adolescent's point of view: Studies of forms in conflict. In N. H. Freeman \& M. V. Cox (Eds.), Visual order (pp. 101-120). Cambridge: Cambridge University Press.

FREEMAN, N. H. (1980). Strategies of representation in young children. New York: Academic Press.

FreEman, N. H. (1986). How should a cube be drawn? British Journal of Developmental Psychology, 4, 317-322.

Freeman, N. H. (1987). Current problems in the development of representational picture-production. Archives de psychologie, 55, 127-152.

GREEN, J. A. (1988). Loglinear analysis of cross-classified ordinal data: Applications in developmental research. Child Development, 59, 1-25.

Hagen, M. A. (1985). There is no development in art. In N. H. Freeman \& M. V. Cox (Eds.), Visual order (pp. 59-77). Cambridge: Cambridge University Press.

Hagen, M. A. (1986). Varieties of realism. Cambridge: Cambridge University Press.

Kubovy, M. (1986). The psychology of perspective and Renaissance art. Cambridge: Cambridge University Press.

MITCHELMORE, M. C. (1987). Why do children not use parallels in their drawings of cubes? Archives de psychologie, 55, 179-194.

MOORE, V. (1987). The influence of experience on children's drawings of a familiar and unfamiliar object. British Journal of Developmental Psychology, 5, 221-239.

Nicholis, A. L., \& KennedY, J. M. (1992). Drawing development: From similarity of features to direction. Child Development, 63, 227-241.

Phillips, W. A., Hobbs, S. B., \& Pratt, F. R. (1978). Intellectual realism in children's drawings of cubes. Cognition, 6, 15-33.

PirenNe, M. H. (1970). Optics, painting and photography. Cambridge: Cambridge University Press.

WhITE, J. (1967). The birth and rebirth of pictorial space. Somerset, U.K.: Butler \& Tanner.

Willats, J. (1981). What do the marks in the picture stand for? The child's acquisition of systems of transformation and denotation. Visual Arts Research, 7, 18-33.

Willats, J. (1984). Getting the drawing to look right as well as to be right: The interaction between production and perception as a mechanism of development. In W. R. Crozier \& A. J. Chapman (Eds.), Cognitive processes in the perception of ant (pp. 111-125). Amsterdam: North-Holland.

(Manuscript received July 10, 1992; revision accepted for publication April 29, 1993.) 